JOURNAL OF SYNCHROTRON RADIATION

ISSN 1600-5775

Received 17 November 2014

Accepted 13 March 2015

Edited by M. Yabashi, RIKEN SPring-8 Center, Japan

\# Currently at CNRS, University of Rennes 1, 263 Avenue General Leclerc, 35042 Rennes, France.

Keywords: FEL; X-ray; pump-probe; time-resolved.

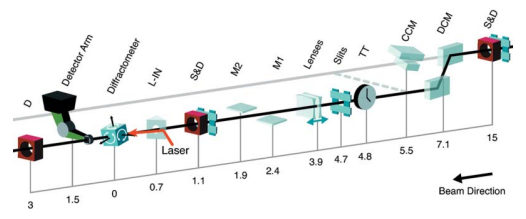

OPEN $\odot$ ACCESS

\section{The X-ray Pump-Probe instrument at the Linac Coherent Light Source}

\author{
Matthieu Chollet, ${ }^{a}$ Roberto Alonso-Mori, ${ }^{a}$ Marco Cammarata, ${ }^{a} \ddagger$ Daniel Damiani, ${ }^{a}$ \\ Jim Defever, a James T. Delor, ${ }^{a}$ Yiping Feng, ${ }^{a}$ James M. Glownia, ${ }^{a}$ J. Brian Langton, ${ }^{a}$ \\ Silke Nelson, ${ }^{a}$ Kelley Ramsey, ${ }^{a}$ Aymeric Robert, ${ }^{a}$ Marcin Sikorski, ${ }^{a}$ Sanghoon Song, ${ }^{a}$ \\ Daniel Stefanescu, ${ }^{a}$ Venkat Srinivasan, ${ }^{a}$ Diling Zhu, ${ }^{a *}$ Henrik T. Lemke ${ }^{b}$ and \\ David M. Fritz ${ }^{\mathrm{a}}$
} aLinac Coherent Light Source, SLAC National Accelerator Laboratory, 2575 Sand Hill Road, Menlo Park, CA 94025, USA,
and ${ }^{\mathbf{b}}$ Paul Scherrer Institut, CH-5232 Villigen, Switzerland. *Correspondence e-mail: dlzhu@slac.stanford.edu

The X-ray Pump-Probe instrument achieves femtosecond time-resolution with hard X-ray methods using a free-electron laser source. It covers a photon energy range of 4-24 keV. A femtosecond optical laser system is available across a broad spectrum of wavelengths for generating transient states of matter. The instrument is designed to emphasize versatility and the scientific goals encompass ultrafast physical, chemical and biological processes involved in the transformation of matter and transfer of energy at the atomic scale.

\section{Introduction}

The Linac Coherent Light Source (LCLS), a US Department of Energy Office of Science user facility operated by Stanford University, achieved first light in 2009 (Emma et al., 2010). The X-ray Pump-Probe (XPP) instrument, located in the third hutch of the Near Experimental Hall (NEH), was the first hard $\mathrm{X}$-ray instrument to receive LCLS hard X-rays and started user operation in 2010. LCLS provides transversely coherent hard X-rays with unprecedented flux and short pulse duration. These characteristics enable the experimental investigation of structural dynamics with X-ray techniques by directly following the time evolution of the electron density during the course of a photo-induced biological, chemical or physical transformation. The XPP instrument predominantly uses ultrafast optical laser pulses to generate transient states of matter. Hard X-ray pulses from the LCLS probe the structural dynamics initiated by the optical excitation. The optical laser has the ability to create or access a wide variety of excited states, and these can be manipulated by changing the laser pulse energy, wavelength, temporal profile and even using various nonlinear excitation mechanisms. X-ray absorption and emission spectroscopy are used to track changes in the electronic and site-specific nuclear structure, and X-ray scattering is the dominant tool for probing concomitant structural changes.

\section{Instrument overview}

The XPP instrument operates in the hard X-ray regime (i.e. above $4 \mathrm{keV}$ ). A summary of the XPP instrument parameters is listed in Table 1. A set of mirrors, consisting of siliconcarbide coated silicon substrates with an incidence angle of $1.32 \mathrm{mrad}$, is located in the front-end enclosure of the $\mathrm{NEH}$ which feeds the hard X-ray beam to all LCLS hard X-ray 
Table 1

X-ray parameters and capabilities of the XPP instrument.

\begin{tabular}{|c|c|}
\hline Instrument name & XPP \\
\hline Mirrors, incidence angle & $2 \times \mathrm{SiC}$ on $\mathrm{Si}, 1.32 \mathrm{mrad}$ \\
\hline Monochromaticity $(\Delta E / E) \dagger$ & $1 \times 10^{-3}(\mathrm{SASE}), 2 \times 10^{-4}$ (seeding) \\
\hline Energy range (keV) & 4 to 11 (fundamental) \\
\hline Unfocused beam size $(\mu \mathrm{m})$ & 500 at $8.3 \mathrm{keV}$ \\
\hline Focused beam size $(\mu \mathrm{m})$ & $2-500$ \\
\hline Focusing capability & Be lenses, 1D and 2D focusing \\
\hline Flux (photons pulse ${ }^{-1}$ ) & $1 \times 10^{12}$ (fundamental $\left.\$\right)$ \\
\hline Pulse length (fs) & $5-200$ \\
\hline Repetition rate & $120,60,30,10,5,1$, on demand \\
\hline Optical laser pulse energy $(\mathrm{mJ})$ & $20(800 \mathrm{~nm}), 4-5(400 \mathrm{~nm}), 1(266 \mathrm{~nm})$ \\
\hline Optical laser pulse width (fs) & $10-150$ \\
\hline Standard detectors & $\begin{array}{l}\text { CSPAD, CSPAD-140K, ePix, Rayonix, } \\
\text { ORCA, PIPS diodes }\end{array}$ \\
\hline Sample environment & $\begin{array}{l}\text { Huber three-circle goniometer, kappa } \\
\text { diffractometer, general purpose vacuum } \\
\text { chamber, liquid jet, He enclosure, } \\
\text { Oxford } \mathrm{LN}_{2} \text { cryojet down to } 100 \mathrm{~K}\end{array}$ \\
\hline
\end{tabular}

$\dagger$ Typical single-shot value. $\ddagger$ Excluding beamline and instrument transmission

hutches. These mirrors also serve to define the maximum photon energy that can be delivered to the LCLS hard X-ray hutches to $25 \mathrm{keV}$. The fundamental photon energy covers the 4-11 keV range; higher photon energies can be reached by using the third harmonic.

The most upstream components of the XPP instrument are located in hutch 2 and consist of slits and diagnostics located $15 \mathrm{~m}$ upstream of the sample as indicated in Fig. 1. The X-ray beam enters hutch 3, the XPP hutch, just before the doublecrystal monochromator, indicated in Fig. 1 by DCM. We now further describe the specifications of the key components of the XPP instrument.

Monochromators. The XPP instrument has two distinct configurations. It can operate in the LCLS main line and can therefore take full advantage of the first-harmonic capabilities of the LCLS (SASE, seeding, two-color, etc.) (Amann et al.,
2012; Lutman et al., 2014). To operate in this configuration, all components located downstream of the dashed line in Fig. 1 are translated into the LCLS main line (indicated by the gray line in Fig. 1). In that configuration a $\mathrm{Si}(111)$ channel-cut monochromator (CCM), operating in vertical scattering geometry (Narayanan et al., 2008), allows a narrower bandwidth $\left(\Delta E / E=1.4 \times 10^{-4}\right)$ to be delivered to experiments that require broad and intensive scans of the incident photon energy.

For experiments that require a monochromatic beam, but do not intend to scan the incident energy significantly, a custom-built (JJ X-ray, Denmark) large-offset DCM (Zhu et al., 2014) is used. In that configuration, all components located downstream of the dashed line are translated $600 \mathrm{~mm}$ horizontally as displayed in Fig. 1. The DCM can either operate with $\mathrm{Si}(111)$ or $\mathrm{C}^{*}(111)$ crystals. When operating with the latter, it not only provides a better energy resolution $(\Delta E / E=$ $5.3 \times 10^{-5}$ ) but most importantly allows for multiplexing with another instrument located downstream in the Far Experimental Hall (Feng et al., 2013; Zhu et al., 2014, 2015). When operating with the $\mathrm{Si}(111)$, only XPP can use the LCLS beam when the crystal is in place. Fortunately, the beam can be rapidly provided to any of the $\mathrm{FEH}$ instruments by translating the first silicon DCM crystal from the beam path.

$X$-ray focusing lenses. The unfocused beam at XPP is typically $0.5 \mathrm{~mm} \times 0.5 \mathrm{~mm}$ in size. Beryllium compound refractive lenses can focus the beam (Snigirev et al., 1996) and thereby provide beam size control in one or two dimensions. The lenses are located $3.85 \mathrm{~m}$ upstream of the sample and can be adjusted $\pm 150 \mathrm{~mm}$ longitudinally. The minimum spot size was measured to be slightly below $2 \mu \mathrm{m}$ (as a result of the finite SASE bandwidth and optics imperfections). An asymmetric focusing option allows a line focus to be produced in the sample plane for applications such as grazing-incidence experiments or elongated samples.

Pulse picker. A fast shutter is available for selecting single X-ray pulses from the LCLS pulse train on demand, as well as for reducing the repetition rate. It consists of a rotating channel whose angle is swung back and forth by a stepper motor to create a brief opening time. It can be used to create arbitrary pulse train time patterns provided that the pulse train structure has an average rate of equal to or less than $10 \mathrm{~Hz}$.

Mirrors. Two silicon mirrors located 1.9 and $2.4 \mathrm{~m}$ upstream of the sample allow the beam to be delivered with a vertical grazing angle (as, for example, required for liquid surfaces). They can also offer the interesting option to reduce the third-harmonic content of the LCLS beam by operating them above the critical angle for these energies. 
Diffractometer. A custom-built (Huber, Germany) diffractometer provides various degrees of freedom together with high load capacity, thus allowing the precise orientation of samples or sample environments such as vacuum chambers, gas/liquid injectors, etc. A kappa diffractometer module is also available. The detector positioning is provided by a robotic arm (Stäubli, Switzerland). It provides a sample-detector distance of up to $1.5 \mathrm{~m}$ and can either operate in cartesian or spherical coordinates once the origin is defined (i.e. sample).

Optical laser system. Core laser systems at the LCLS consist of an ultrashort-pulse Ti:sapphire oscillator synchronized to the FEL seeding a commercially available chirped pulse amplifier producing $4 \mathrm{~mJ}$ at $40 \mathrm{fs}$. An additional home-built four-pass amplifier can boost the pulse energy to over $30 \mathrm{~mJ}$ to most of the experimental hutches. Wavelength conversion inside the experimental hutches can cover a broad spectral range from $200 \mathrm{~nm}$ to $150 \mu \mathrm{m}$. A more in-depth description of the optical laser capabilities at LCLS is given by Minitti et al. (2015).

Timing diagnostics. Typical phase locking between the accelerator and the laser system only hold the temporal jitter between the two sources to about $200 \mathrm{fs}$ full width at halfmaximum (FWHM). In order to take full advantage of the short pulses and reach pulse-length-limited time resolution, diagnostics that measure the relative arrival time between laser and X-ray pulses have been developed. After demonstrating both a spatial and spectral approach for such a diagnostics for hard X-rays at XPP (Harmand et al., 2013), the spectral method was implemented into a standard beamline diagnostics (Bionta et al., 2011; Lemke et al., 2013). It is based on the X-ray induced change in refractive index of a thin target probed by a chirped broadband white-light continuum pulse derived from the optical laser. The X-ray induced change of optical light transmission can be resolved in an optical spectrometer for each pulse. Target materials used are typically either silicon nitride $\left(\mathrm{Si}_{3} \mathrm{~N}_{4}\right)$ or Ce:YAG crystals of different thicknesses, to accommodate for different beam conditions.

Additional diagnostics. The self-amplified spontaneous emission (SASE) process induces pulse-to-pulse fluctuations of the beam properties, such as pulse energy, duration, spatial profile, wavefront, temporal profile and spectral content. In situ pulse property monitoring is thus crucial for data interpretation. Multiple intensity-position monitors (Feng et al., 2011) are installed at various locations along the instrument for pulse-to-pulse intensity normalization. The spatial profile of the LCLS beam can be also measured at various locations along the XPP instrument using scintillating screens with high-resolution camera-lens combinations.

Spectrometers. The spectral intensity profile can be measured on every shot by means of a thin (bent) crystal transmissive spectrometer (Zhu et al., 2013). This device has an energy range that covers the full X-ray FEL spectrum and sufficient resolution to resolve individual SASE spikes. A multi-crystal wavelength-dispersive X-ray emission spectrometer based on the von Hamos geometry and a monochromatic analyser crystal based on the Rowland geometry are also available for photon-in photon-out spectroscopic measurements (Alonso-Mori et al., 2012).

Detectors. Several X-ray detectors are available and integrated with the XPP data acquisition system: the 1 Mpixel CSPAD, the 140 kpixel CSPAD-140k (Herrmann et al., 2013), a Rayonix MX170-HS detector, and the ePix series detector under development (Dragone et al., 2014). For more information about the LCLS detectors see Blaj et al. (2015).

\section{Highlights}

The XPP instrument has been used in a wide range of scientific investigations ranging from materials science, chemistry, nonlinear optics and protein crystallography. The following examples illustrate some capabilities of the instrument.

\subsection{Femtosecond diffraction}

Femtosecond diffraction has been envisioned as one of the primary measurement techniques at the XPP instrument to elucidate complex interactions and correlations in materials via the study of transient atomic scale structural dynamics generated by ultrafast excitations. For example, Beaud et al. (2014) observed the ultrafast melting of the charge and orbitally ordered phase in an epitaxically grown perovskite manganite film $\left[\mathrm{Pr}_{0.5} \mathrm{Ca}_{0.5} \mathrm{MnO}_{3}\right.$ (PCMO)]. The film was pumped with a $55 \mathrm{fs}, 800 \mathrm{~nm}$ optical pulse focused down to $230 \mu \mathrm{m} \times 230 \mu \mathrm{m}$. By taking advantage of the intense and short X-ray pulses, different superlattice reflections, each sensitive to different components of the phase transition, were measured.

Reflections $(0 k / 20)$ are sensitive to the orbital order and Jahn-Teller distortion, while $\left(\begin{array}{lll}0 & k & 0\end{array}\right)$ reflections are more sensitive to the charge order. Reflections $(h k / 2 l)$ give information about the overall structural distortion. Fig. 2 shows the observed time-resolved signal for different superlattice reflections at a pump fluence of $2.7 \mathrm{~mJ} \mathrm{~cm}^{-2}$. The observed

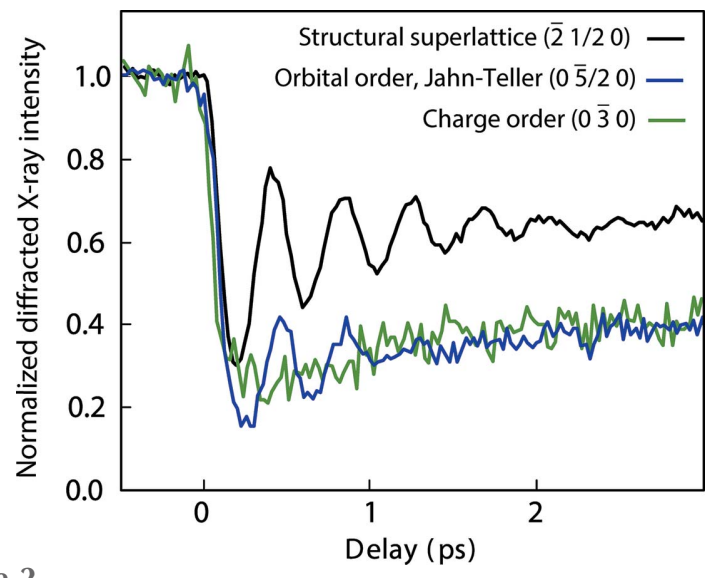

Figure 2

Time-resolved normalized diffracted X-ray intensity for three superlattice reflections. The $(-21 / 20)$ reflection is measured off resonance at $6.53 \mathrm{keV}$. The $(0-5 / 20)$ reflection is measured near resonance at $6.553 \mathrm{keV}$, and the $(0-30)$ reflection is measured on resonance at $6.555 \mathrm{keV}$. [Reprinted by permission from Macmillan Publishers Ltd: Beaudet al. (2014). Nat. Mater. 13, 923-927, copyright (2014).] 
dynamics are dominated by a $2.45 \mathrm{THz}$ oscillation which is characteristic of a coherent optical phonon mode in those type of materials when excited with short optical pulses (Matsuzaki et al., 2009).

In another experiment using femtosecond diffraction, Först et al. (2013) and Mankowsky et al. (2014) demonstrated the potential of using photoexcitation to drive phase transitions in strongly correlated material systems. More generally, the ability to measure lattice dynamics with femtosecond X-rays opens the way towards better understanding and control of ultrafast phase transitions.

\subsection{Femtochemistry}

Femtochemistry of molecular sample systems in solution is another field of research present at the XPP instrument. The ultrafast timescales accessible at LCLS allow for the first time to investigate chemical processes like charge transfer, bond cleavage and molecular vibrations in a solvated environment. These processes are highly relevant for applied chemical or biological processes. A large interest is devoted to molecular photocatalysts due to their potential for charge separation, which can technically be exploited for energy conversion from optical light. In that context, dissolved iron(II) tris(2,2'bipyridine) $\left(\left[\mathrm{Fe}^{\mathrm{II}}(\mathrm{bpy})_{3}\right]^{2+}\right)$ has been studied intensively at XPP, as a model system for abundant transition metal complexes. Here, an optically excited metal-to-ligand charge transfer (MLCT) state is depleted within less than 200 fs to a high-spin (HS) state, which has been previously extensively studied by optical and X-ray methods (Chergui, 2013; Canton et al., 2014; Gawelda et al., 2007; Bressler et al., 2009).

Optically induced local structural changes around the Fe center of the molecule were studied through changes in the Fe $K$-edge X-ray absorption near-edge structure (XANES) (Lemke et al., 2013). An aqueous $\left[\mathrm{Fe}^{\mathrm{II}}(\mathrm{bpy})_{3}\right]^{2+}$ solution in a liquid sheet jet was excited to the MLCT state and the transition cascade into the HS state consequently probed by changes to the absorption cross section of monochromatic $\mathrm{X}$-rays. The intense FEL pulses allowed the entire transient XANES spectra to be scanned in minutes with sufficient statistics to resolve the transient changes. The observed pump-probe difference spectra confirmed picosecond results from the HS state recorded at a synchrotron source (Gawelda et al., 2007), while at short time delays a hint of a different spectral component, potentially the MLCT state, was observed. The ultrafast rise of the HS state XANES signals could for the first time be characterized (Fig. 3a). More detailed information about the transient electronic transitions in $\left[\mathrm{Fe}^{\mathrm{II}}(\mathrm{bpy})_{3}\right]^{2+}$ were obtained by X-ray emission spectroscopy (Zhang et al., 2014).

Fluorescence lines from occupied electron levels to core holes created by the X-rays contain information about the chemically active electron configurations. In iron, the $K \beta_{1,3}$ emission line reflects changes of the Fe $3 d$ electron spin state through interaction of $3 d$ and $3 p$ electrons (Glatzel \& Bergmann, 2005) while it remains less sensitive to changes of the chemical configuration (Lee et al., 2010). Transient combina-

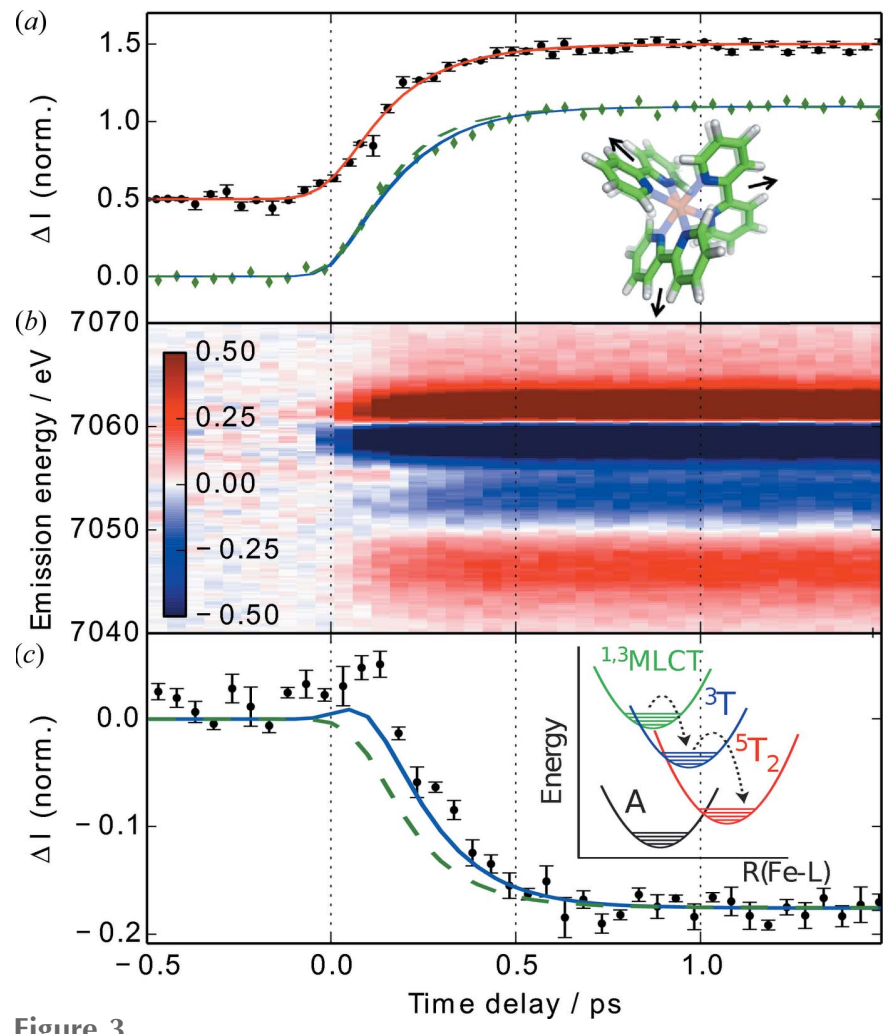

Figure 3

Spectroscopic pump-probe results from the photoexcited spin transition of $\left[\mathrm{Fe}^{\mathrm{II}}(\mathrm{bpy})_{3}\right]^{2+}$ in solution. After excitation into the MLCT state, transition into the $\mathrm{HS}^{5} \mathrm{~T}_{2}$ state [inset $(c)$ ] causes an increase of the Fe-toligand distance [inset $(a)$ ]. This structural change is tracked by the XANES trace at $7125 \mathrm{eV}[(a)$, black symbols, vertically offset by 0.5$]$. The structural HS rise has been fitted by an exponential rise of $162 \pm 6 \mathrm{fs}$ (Lemke et al., 2013). (b) Time-dependent $K \beta$ emission difference spectra. The transient traces at $7061 \mathrm{eV}$ and $7054 \mathrm{eV}$ have been extracted [( $a)$ green diamond symbols and (c) black symbols, respectively]. The data have been overlaid with results from global fits including (blue lines) and excluding (green dashed lines) population of and intermediate ${ }^{3} \mathrm{~T}$ triplet state (Zhang et al., 2014). [Reprinted by permission from Macmillan Publishers Ltd: Zhang et al. (2014). Nature (London), 509, 345-348, copyright (2014).]

tions of spin state can therefore be interpreted through ground-state reference spectra. Reference samples with similar coordination bonding were chosen in order to account for the remaining covalent content in the $K \beta_{1,3}$ spectra. An energy-dispersive von Hamos spectrometer populated with four cylindrically bent $\mathrm{Ge}(620)$ crystal analyzers (AlonsoMori et al., 2012) was used to measure the entire $K \beta_{1,3}$ line with a CSPAD-140K area detector. The changes in the emission lines were recorded and sorted as a function of pumpprobe time delay (Fig. $3 b$ ). Analysis using reference spectra revealed experimental proof for population of $\mathrm{a}^{3} \mathrm{~T}$ triplet state as intermediate state between MLCT and HS state (Fig. $3 c$ ), which had previously been debated on the basis of ultrafast XANES measurements (Bressler et al., 2009).

\section{Conclusion}

The XPP instrument is a versatile tool for performing pumpprobe experiments in the hard X-ray regime. The combination 
of the large number of photons per pulse and the temporal resolution characteristic of LCLS allows for investigation of ultrafast physical, chemical and biological processes. X-ray scattering, absorption and emission spectroscopy are some of the dominant techniques used at the XPP instrument for probing structural and electronic changes. More details about the XPP instrument can be found on the following website: http://lcls.slac.stanford.edu/xpp.

\section{Facility access}

LCLS instruments are open to academia, industry, government agencies and research institutes worldwide for scientific investigations. There are two calls for proposals per year and an external peer-review committee evaluates proposals based on scientific merit and instrument suitability. Access is without charge for users who intend to publish their results. Prospective users are encouraged to contact instrument staff members to learn more about the science and capabilities of the facility, and opportunities for collaboration.

\section{Acknowledgements}

We thank Terry Anderson and Gregory Stewart for helping with the figures. Portions of this research were carried out at the Linac Coherent Light Source (LCLS) at the SLAC National Accelerator Laboratory. LCLS is an Office of Science User Facility operated for the US Department of Energy Office of Science by Stanford University.

\section{References}

Alonso-Mori, R., Kern, J., Sokaras, D., Weng, T.-C., Nordlund, D., Tran, R., Montanez, P., Delor, J., Yachandra, V. K., Yano, J. \& Bergmann, U. (2012). Rev. Sci. Instrum. 83, 073114.

Amann, J. et al. (2012). Nat. Photon. 6, 693-698.

Beaud, P. et al. (2014). Nat. Mater. 13, 923-927.

Bionta, M. R., Lemke, H. T., Cryan, J. P., Glownia, J. M., Bostedt, C., Cammarata, M., Castagna, J.-C., Ding, Y., Fritz, D. M., Fry, A. R., Krzywinski, J., Messerschmidt, M., Schorb, S., Swiggers, M. L. \& Coffee, R. N. (2011). Opt. Express, 19, 21855-21865.

Blaj, G., Caragiulo, P., Carini, G., Carron, S., Dragone, A., Freitag, D., Haller, G., Hart, P., Hasi, J., Herbst, R., Herrmann, S., Kenney, C., Markovic, B., Nishimura, K., Osier, S., Pines, J., Reese, B., Segal, J., Tomada, A. \& Weaver, M. (2015). J. Synchrotron Rad. 22, 577583.

Bressler, C., Milne, C., Pham, V.-T., Elnahhas, A., van der Veen, R. M., Gawelda, W., Johnson, S., Beaud, P., Grolimund, D., Kaiser, M., Borca, C. N., Ingold, G., Abela, R. \& Chergui, M. (2009). Science, 323, 489-492.

Canton, S. E., Zhang, X., Lawson Daku, L. M., Smeigh, A. L., Zhang, J., Liu, Y., Wallentin, C.-J., Attenkofer, K., Jennings, G., Kurtz, C. A., Gosztola, D., Wärnmark, K., Hauser, A. \& Sundström, V. (2014). J. Phys. Chem. C, 118, 4536-4545.

Chergui, M. (2013). Spin-Crossover Materials: Properties and Applications, edited by M. A. Halcrow, ch. 15, pp. 405-424. New York: John Wiley and Sons.
Dragone, A., Caragiulo, P., Markovic, B., Herbst, R., Reese, B., Herrmann, S., Hart, P., Segal, J., Carini, G., Kenney, C. \& Haller, G. (2014). J. Phys. Conf. Ser. 493, 012012.

Emma, P. et al. (2010). Nat. Photon. 4, 641-647.

Feng, Y., Alonso-Mori, R., Barends, T., Blank, V., Botha, S., Chollet, M., Damiani, D. S., Doak, R. B., Glownia, J. M., Koglin, J. M., Lemke, H. T., Messerschmidt, M., Nass, K., Nelson, S., Schlichting, I., Shoeman, R. L., Shvyd'ko, Y. V., Sikorski, M., Song, S., Stoupin, S., Terentyev, S., Williams, G. J., Zhu, D., Robert, A. \& Boutet, S. (2015). J. Synchrotron Rad. 22, 626-633.

Feng, Y., Feldkamp, J. M., Fritz, D. M., Cammarata, M., Aymeric, R., Caronna, C., Lemke, H. T., Zhu, D., Lee, S., Boutet, S., Williams, G., Tono, K., Yabashi, M. \& Hastings, J. B. (2011). Proc. SPIE, 8140, 81400Q.

Feng, Y. et al. (2013). Proc. SPIE, 8778, 87780B.

Först, M., Mankowsky, R., Bromberger, H., Fritz, D. M., Lemke, H., Zhu, D., Chollet, M., Tomioka, Y., Tokura, Y., Merlin, R., Hill, J. P., Johnson, S. L. \& Cavalleri, A. (2013). Solid State Commun. 169, 24 27.

Gawelda, W., Pham, V.-T., Benfatto, M., Zaushitsyn, Y., Kaiser, M., Grolimund, D., Johnson, S., Abela, R., Hauser, A., Bressler, C. \& Chergui, M. (2007). Phys. Rev. Lett. 98, 057401.

Glatzel, P. \& Bergmann, U. (2005). Coord. Chem. Rev. 249, 65-95.

Harmand, M., Coffee, R., Bionta, M. R., Chollet, M., French, D., Zhu, D., Fritz, D. M., Lemke, H. T., Medvedev, N., Ziaja, B., Toleikis, S. \& Cammarata, M. (2013). Nat. Photon. 7, 215-218.

Herrmann, S., Boutet, S., Duda, B., Fritz, D., Haller, G., Hart, P., Herbst, R., Kenney, C., Lemke, H., Messerschmidt, M., Pines, J., Robert, A., Sikorski, M. \& Williams, G. (2013). Nucl. Instrum. Methods Phys. Res. A, 718, 550-553.

Lee, N., Petrenko, T., Bergmann, U., Neese, F. \& DeBeer, S. (2010). J. Am. Chem. Soc. 132, 9715-9727.

Lemke, H. T., Bressler, C., Chen, L. X., Fritz, D. M., Gaffney, K. J., Galler, A., Gawelda, W., Haldrup, K., Hartsock, R. W., Ihee, H., Kim, J., Kim, K. H., Lee, J. H., Nielsen, M. M., Stickrath, A. B., Zhang, W., Zhu, D. \& Cammarata, M. (2013). J. Phys. Chem. A, 117, 735-740.

Lutman, A. A., Decker, F.-J., Arthur, J., Chollet, M., Feng, Y., Hastings, J., Huang, Z., Lemke, H., Nuhn, H.-D., Marinelli, A., Turner, J. L., Wakatsuki, S., Welch, J. \& Zhu, D. (2014). Phys. Rev. Lett. 113, 254801.

Mankowsky, R., Subedi, A., Först, M., Mariager, S. O., Chollet, M., Lemke, H., Robinson, J., Glownia, J., Minitti, M., Frano, A., Fechner, M., Spaldin, N. A., Loew, T., Keimer, B., Georges, A. \& Cavalleri, A. (2014). Nature (London). 516, 71-73.

Matsuzaki, H., Uemura, H., Matsubara, M., Kimura, T., Tokura, Y. \& Okamoto, H. (2009). Phys. Rev. B, 79, 235131.

Minitti, M. P., Robinson, J. S., Coffee, R. N., Edstrom, S., Gilevich, S., Glownia, J. M., Granados, E., Hering, P., Hoffmann, M. C., Miahnahri, A., Milathianaki, D., Polzin, W., Ratner, D., Tavella, F., Vetter, S., Welch, M., White, W. E. \& Fry, A. R. (2015). J. Synchrotron Rad. 22, 526-531.

Narayanan, S., Sandy, A., Shu, D., Sprung, M., Preissner, C. \& Sullivan, J. (2008). J. Synchrotron Rad. 15, 12-18.

Snigirev, A., Kohn, V., Snigireva, I. \& Lengeler, B. (1996). Nature (London), 384, 49-51.

Zhang, W. et al. (2014). Nature (London), 509, 345-348.

Zhu, D., Cammarata, M., Feldkamp, J., Fritz, D., Hastings, J., Lee, S., Lemke, H., Robert, A., Turner, J. \& Feng, Y. (2013). J. Phys. Conf. Ser. 425, 052033.

Zhu, D., Feng, Y., Stoupin, S., Terentyev, S. A., Lemke, H. T., Fritz, D. M., Chollet, M., Glownia, J. M., Alonso-Mori, R., Sikorski, M., Song, S., van Driel, T. B., Williams, G. J., Messerschmidt, M., Boutet, S., Blank, V. D., Shvyd'ko, Y. V. \& Robert, A. (2014). Rev. Sci. Instrum. 85, 063106. 